\title{
Empyema of Gall Bladder- Typhoid
}

\author{
V. K. Vineeth ${ }^{1}$, Jagadeesh Chandrasekaran ${ }^{2}$, Aarthee Asokan ${ }^{3}$, \\ P. Senthur Nambi ${ }^{4}$ \\ ${ }^{1}$ FNB Registrar Infectious Diseases, Department of Infectious Diseases, Apollo Main Hospitals, Greams Lane \\ Off Greams Road, Chennai, Tamilnadu 600060, India. \\ ${ }^{2}$ Senior Consultant, Department of Internal Medicine, Apollo Main Hospitals, Greams Lane Off Greams Road, \\ Chennai, Tamilnadu 600060, India. \\ ${ }^{3}$ DNB Resident, Department of Internal Medicine, Apollo Main Hospitals, Greams Lane Off Greams Road, \\ Chennai, Tamilnadu 600060, India. \\ ${ }^{4}$ Senior Consultant, Department of Infectious Diseases, Apollo Main Hospitals, Greams Lane Off Greams Road, \\ Chennai, Tamilnadu 600060, India. \\ Corresponding Author: V. K. Vineeth
}

\begin{abstract}
Enteric fever involves reticuloendothelial system. Most common complications are intestinal perforation, hepatitis, encephalopathy and bleeding ileal ulcers. Apart from known complications we present an interesting case of middle aged female with uncontrolled diabetes presenting with empyema of gall bladder secondary to Salmonella typhi. Both blood and gallbladder tissue bacteriological cultures had Salmonella typhi confirming our diagnosis. It was noteworthy that empyema was found intraoperatively. Patient recovered after cholecystectomy and antibiotics. Considering this rare complication we advise Typhoid conjugate vaccine in tropical countries. This case report paves way to recognize this complication to watch for.
\end{abstract}

Key Words: Enteric fever, Empyema, complications, Typhoid vaccine, gall bladder

\section{INTRODUCTION}

Enteric fever is a common in tropical countries. It is caused mainly by Salmonella typhi and accounts for nearly 27 million cases worldwide and 200,000 deaths annually ${ }^{(1)}$. Enteric fever involves the reticuloendothelial system such as bone marrow, spleen, and liver. Due to involvement of Peyer's patches of terminal ileum, perforation of bowel is a known complication. However, gallbladder perforation and empyema are rare.

It is most often found intraoperatively and empyema cannot be diagnosed through routine imaging. Most common organisms isolated are Klebsiella, Bacteroides, and E. coli. Usually patients with diabetes, patients on immunosuppressants and hemoglobinopathy are at a higher risk of developing empyema. Most common pathogenesis regarding empyema is stasis, ischaemia and cystic duct obstruction $^{(2)}$.

We present a middle aged female diabetic with empyema of gall bladder caused by a rare pathogen.

\section{CASE HISTORY}

39 Year old female poorly controlled diabetic with recent $\mathrm{HbA} 1 \mathrm{c}$ of 8.8 came with complaints of fatigue, low grade fever since 8 days and abdominal pain of 6 days duration. She had skipped her antidiabetic drugs since 3 weeks. On examination her pulse rate was $100 / \mathrm{min}$, BP-160/100mm of $\mathrm{Hg}$, systemic examination showed diffuse tenderness in the abdomen.

Initial blood work up showed neutrophilic leukocytosis, normal platelets, normal serum ketones, normal amylase and 
lipase and mild transaminitis. Her urine routine did not show pyuria.

TABLE 1: INVESTIGATIONS

\begin{tabular}{|l|l|}
\hline Investigation (first 24 hours) & values \\
\hline $\mathrm{Hb}$ & 12.1 \\
\hline Total count & $171300 / \mathrm{mm}^{3}$ \\
\hline Differential count & $\begin{array}{l}80 \% \text { neutrophil, 10\% } \\
\text { 1ymphocytes, 10\% monocytes }\end{array}$ \\
\hline Platelet count & $2,45,000 / \mathrm{mm}^{3}$ \\
\hline Creatinine & $0.8 \mathrm{mg} / \mathrm{dl}$ \\
\hline Total bilirubin & 0.8 \\
\hline Direct bilirubin & 0.6 \\
\hline Indirect bilirubin & 0.2 \\
\hline SGOT & $52 \mathrm{U} / \mathrm{L}$ \\
\hline SGPT & $57 \mathrm{U} / \mathrm{L}$ \\
\hline ALP & $126 \mathrm{U} / \mathrm{L}$ \\
\hline GGT & $90 \mathrm{U} / \mathrm{L}$ \\
\hline Hba1c & 8.2 \\
\hline Lipase & $96 \mathrm{U} / \mathrm{L}$ \\
\hline Amylase & $70 \mathrm{U} / \mathrm{L}$ \\
\hline &
\end{tabular}

Considering sepsis syndromecommunity acquired with probable foci in abdomen, blood was drawn for cultures and was started on empirical broad spectrum antibiotic of Meropenem. Screening of community acquired febrile illness like malaria, dengue, leptospirosis were negative. Transthoracic echocardiography was normal.

Ultrasound imaging showed features of calculous cholecystitis. On contrast enhanced CT to look for complications involving gall bladder showed sludge with multiple intraluminal calculi measuring about $1 \mathrm{cms}$ in gall bladder with associated wall thickening and enhancement with focal areas of wall disruption near fundus and lateral wall with minimal pericholecystic fluid and surrounding fat stranding suggesting of acute calculus gangrenous cholecystitis.

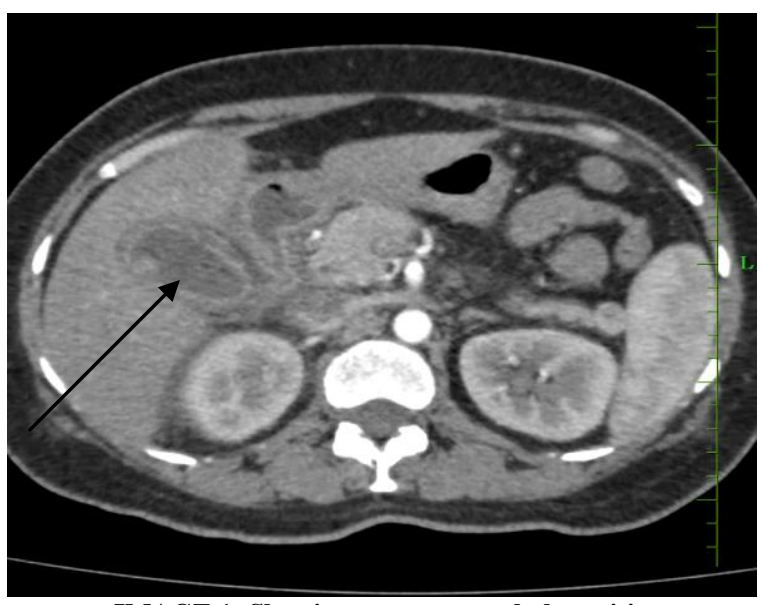

IMAGE 1: Showing gangrenous cholecystitis
Considering this scenario patient was taken up for emergency laparoscopic cholecystectomy.

observations were

- Thick congested gall bladder wall

- Multiple pigmented calculi in gall bladder

- Pus in the gall bladder

- Adhesions in the omentum and hepatic flexure colon

Tissue was sent for histopathology and bacterial culture. In the mean time her blood culture taken in 2 different sets (4 bottles) grew Salmonella typhi sensitive to ceftriaxone, azithromycin, co-trimoxazole and intermediate to ciprofloxacin. Antibiotics were de escalated to azithromycin and ceftriaxone. Patient was better post operatively with stable haemodynamics, settling leukocytosis, well controlled sugars and asymptomatic. On following up, tissue culture had Salmonella typhi with the same sensitivity pattern as blood confirming the source of infection. Histopathology showed wall of gall bladder with transmural necrosis, subacute inflammation with adherent calcareous material.

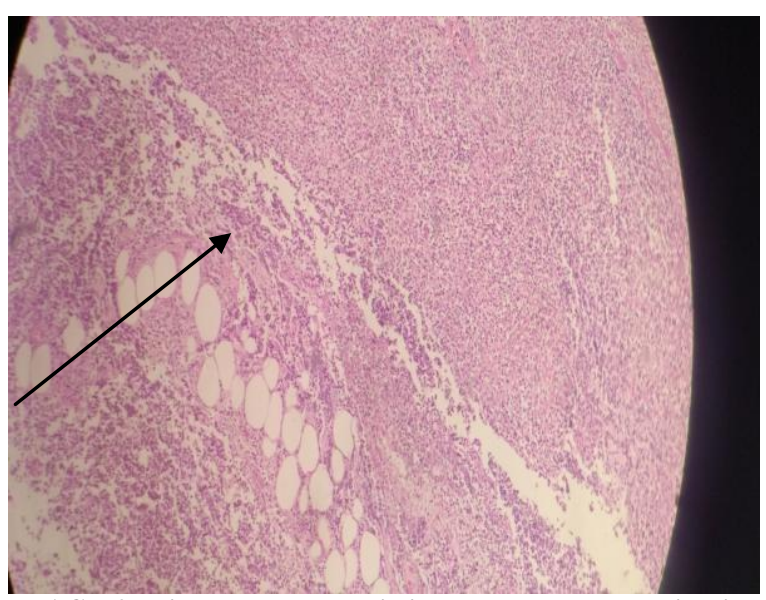

IMAGE 2: Histopathology depicting transmural necrosis with subacute inflammation.

Patient received 14 days of antibiotic therapy. She was doing well in her follow up and was advised typhoid vaccination.

\section{DISCUSSION}

Empyema of gall bladder is not an uncommon complication of acute 
cholecystitis. This complication is usually considered as progression caused due to stasis of bile and cystic duct obstruction. Surprisingly our case did not have any of those. This patient had calculi size less than $2 \mathrm{~cm}$ as mostly duct obstruction are caused large calculi.

As per literature not many cases are report of empyema of gall bladder secondary to Salmonella typhi with bacteraemia.

One of the key risk factor our patient had was uncontrolled diabetes and inadequate drug compliances predisposing her for infections.

In a study titled gallbladder perforation secondary to enteric fever by $\mathrm{N}$ Malik et al ${ }^{(1)}$ concluded that patients with signs and symptoms of typhoid fever along with enteric perforation, gall bladder perforation should be carefully looked for. Typhoid was diagnosed based on IgG and IgM Typhoid tests and the patient did not have bacteraemia. Compared to this our case did not have perforation and empyema was intraoperative finding similarly. Cholecystectomy is the treatment of choice for perforation and empyema.

In a study titled acute acalculous cholecystitis with empyema due to Salmonellosis by D Lianos et $\mathrm{al}^{(2)}$ explained the importance of diagnosing the rare complications of acalculous cholecystitis like empyema, gangrene and perforation. Patient in this study was not bacteraemic, although tissue and stool grew Salmonella sensitive to ciprofloxacin. In contrary our case had bacteraemia with calculous cholecystitis secondary to Salmonella typhi.

A study titled Complications and mortality of typhoid fever: A global systematic review and meta- analysis by Marchello et $\mathrm{al}^{(3)}$ listed typhoid complications which included intestinal perforation, gastrointestinal hemorrhage, hepatitis, cholecystitis, myocarditis, encephalopathy. It is worth observing that empyema was not included in the list of significant complication encountered. So our case paves the way to recognize empyema of gall bladder as one of the complication due to typhoid.

In a similar study by Yousef Khan et al (4) titled acute acalculous cholecystitis complicating typhoid fever in an adult patient with similar presentation and Salmonella enteric isolated from blood. Patient did not undergo surgery as there were no red flag signs of complication and was treated with 2 weeks of ceftriaxone.

Regarding optimum therapeutic options in a study by Veeraraghavan et al ${ }^{(5)}$ titled Typhoid fever: issues in laboratory detection treatment options and concerns in management in developing countries found that reduced fluoroquinolones susceptibility of Salmonella enteric serovar typhi is on rise and is significant concern in developing countries. They advised therapy with third generation cephalosporin and azithromycin. It is noteworthy to observe that in our patient ciprofloxacin had intermediate sensitivity.

\section{CONCLUSION}

This is one of the rare case reports of empyema of gall bladder secondary to Salmonella typhi bacteraemia. We suggest recognizing empyema of gall bladder to be considered as serious complication for adequate quality medical care. Considering the impact of Typhoid on mortality and morbidity we advise administering Typhoid conjugate vaccine in tropical countries. Timely recognizing early and late complications and tackling is the key.

\section{Acknowledgement: None}

\section{Conflict of Interest: None}

\section{Source of Funding: None}

\section{REFERENCES}

1. Malik M N, Mahmood T, Tameez Ud Din A, et al,Gallbladder Perforation Secondary to Enteric Fever: An Interesting Case of Acute Abdomen, Cureus 2019,11(4)e4516.

2. Georgios D Lianos, Panagiota Drosou et al, Acute Acalculous cholecystitis with Empyema due to Salmonellosis, Hindawi 
Case reports in Gastrointestinal medicine, Volume 2019, Article ID 5185314.

3. Christian S Marchello, Megan Birkhold and John A Crump, Complications and mortality of typhoid fever: A global systematic review and meta-analysis, The Journal of Infection 2020 December, 81(6)902-910.

4. Fahim khan, Elhadi B Elouzi, Mohammed Asif, Acute acalculous cholecystitis complicating typhoid fever in an adult patient,Travel Medicine and Infectious diseases,August 2009,7(4):203-6
5. Balaji Veeraraghavan, Agila K Pragasam and Ravikar Ralph, Typhoid fever: Issues in laboratory detection, treatment options and concerns in management in developing countries, Future Science OA 2018 04(06)

How to cite this article: Vineeth VK, Chandrasekaran J, Asokan A et.al. Empyema of gall bladder- typhoid. Int J Health Sci Res. 2021; 11(8): 194-197. DOI: https://doi.org/10. 52403/ijhsr.20210828 